\title{
Adultez en la escuela: notas para su problematización
}

\section{Adulthood in school: some notes for its problematization}

\author{
Klaudio Duarte Quapper I Herman Pezo Hoces \\ Núcleo de Investigación y Acción en Juventudes Universidad de Chile
}

\section{RESUMEN}

En el presente texto problematizamos un conjunto de elementos que surgen desde la discusión bibliográfica actualizada y la observación sistemática de nuestras experiencias de acción directa en comunidades educativas. Nuestra entrada a esas comunidades educativas ha sido a través de la preocupación por las realidades de las y los jóvenes presentes en ellas; sin embargo, un aprendizaje de los últimos años es que uno de los asuntos que requieren ser cuestionados en esos entramados educativos es la construcción de la adultez que ahí se hace. La perspectiva generacional que usamos nos ha abierto interrogantes respecto de la relevancia de agudizar nuestra mirada analítica sobre esta condición de adultez. Los resultados de este ejercicio de problematización nos dan pistas para proponer líneas de investigación. La metodología que usamos es coherente con la sociopraxis, en tanto se produce desde la reflexión sistemática y crítica de nuestra experiencia en las comunidades educativas.

Palabras clave: adultez, perspectiva generacional, relaciones generacionales en la escuela

\section{Contacto:}

Claudio Duarte | Universidad de Chile | claudioduarte@uchile.cl

Herman Pezo | Universidad de Chile I hermanfabian.pezo@gmail.com 


\begin{abstract}
In the present text, we problematize a set of elements arisen from the updated bibliographic discussion and the systematic observation of our direct action experiences in educational communities. Our gateway to those educational communities is based on our concern about the realities of the male and female youngsters present on them. However, a lesson learned in recent years is the need of questioning one of the issues in these educational frameworks: the construction of adulthood developed within them. The generational perspective we used has created questions about the relevance of sharpening our analytical look on this adulthood condition. The results of this problematization provide clues to establish lines of research. The methodology used is consistent with the sociopraxis, while it is generated from the systematic and critical reflection from our experience in educational communities.
\end{abstract}

Keywords: adulthood, generational perspective, generational relationships at school

\title{
INTRODUCCIÓN
}

La adultez goza en nuestras sociedades contemporáneas de un alto prestigio. Por lo mismo, casi no ha sido un asunto de interés para las ciencias de la educación ni para las ciencias sociales. Existe muy poca producción sistemática de conocimientos que colabore a una comprensión de lo que implica su condición de superioridad naturalizada en el orden adultocéntrico de las sociedades actuales, ni para develar los mecanismos que se utilizan en su construcción social y en su acrítica reproducción, y menos para los efectos que estos procesos tienen en las cotidianidades educativas, en las relaciones que las y los diversos actores despliegan en la escuela, ni en las formas en que se dan sus dinámicas organizacionales.

Por ello, en este texto nos proponemos realizar una contribución en la búsqueda de aperturas analíticas sobre la condición de adultez en la época actual. Pretendemos aportar con una reflexión crítica 
sobre las incidencias que tiene en la escuela este (no) tratamiento de lo adulto como un modo de relación social que cuenta con legitimidad inmanente y que reproduce un orden asimétrico entre generaciones. Lo hacemos a través de una reflexión en que problematizamos un conjunto de elementos que surgen desde la discusión bibliográfica actualizada y la observación sistemática de nuestras experiencias de acción directa en comunidades educativas, a partir del trabajo que el Núcleo de Investigación y Acción en Juventudes ha venido realizando por casi dos décadas. En especial, desde el año 2015 hemos desplegado procesos en liceos del país (regiones de Arica y Parinacota, Tarapacá, Coquimbo, Valparaíso, de O'Higgins y Metroplitana) centrados en las preguntas por las condiciones institucionales para la promoción de la participación juvenil-estudiantil y los diálogos intergeneracionales en las comunidades educativas.

Tal como puede intuirse, nuestra entrada o vínculo a esas comunidades educativas ha sido a través de la preocupación por las realidades de las y los jóvenes presentes en ellas. Sin embargo, un aprendizaje de los últimos ańos es que uno de los asuntos que requieren ser cuestionados en esos entramados educativos es la construcción de la adultez que se hace en ellas. La perspectiva generacional que usamos -en vinculación caleidoscópica con clase, género, raza y territorio- nos ha abierto interrogantes sobre las relevancias políticas, teóricas y de método de agudizar nuestra mirada analítica sobre esta condición de adultez.

Los resultados de este primer ejercicio de problematización, como fundamentación inicial de líneas de investigación en este asunto, están contenidos en este texto en cuatro apartados. En el primero, a partir de nuestras observaciones de campo, ponemos de relieve la característica de relaciones generacionales que se dan al interior de las comunidades educativas, con una adultez que no es cuestionada en ellas. En el segundo, desde la revisión bibliográfica, evidenciamos cuáles han sido las dos perspectivas principales en el tratamiento de la adultez desde las escasas investigaciones sociales que actualmente existen en esta materia. Posteriormente, en el apartado tres, decantamos 
la problematización hacia el eje de la escuela como una institución social donde existen personas adultas, que juegan roles decisorios, pero en ella no se reconoce esta adultez. Finalmente, en el cierre de este texto se ofrecen aperturas reflexivas en que proponemos tres líneas de trabajo que podrían, en el tiempo, constituirse como un programa investigativo sobre la adultez con perspectiva generacional.

\section{LA COMUNIDAD EDUCATIVA COMO ESPACIO DE RELACIONES GENERACIONALES Y DE UNA ADULTEZ NO CUESTIONADA}

En nuestra sociedad contemporánea, las ciencias sociales y las ciencias educativas han venido proporcionando un conjunto de perspectivas para observar críticamente las diversas dinámicas que constituyen esta sociedad. Al uso ya tradicional de las condiciones y conflictos de clase para analizar lo social, se sumaron hace décadas los ejes de género y raza, cuestión en que las perspectivas feministas interseccionales han aportado de forma sustantiva (Viveros, 2016). Sin embargo, si nos hacemos cargo de la noción de que el entramado social es complejo, necesitamos la consideración de otras perspectivas que, en la dirección de las ya planteadas, contribuyan a profundizar en esas observaciones críticas de lo social. Una de ellas es considerar las cuestiones territoriales, en que las dinámicas de segregación permiten producir interesantes cuestionamientos respecto de las lógicas y modos en que se dan los procesos de inclusión/exclusión. De forma similar, se ha venido sugiriendo la necesidad de considerar la perspectiva generacional para la comprensión de estas dinámicas sociales (Álvarez, 2018; Duarte, 2018; Ghiardo, 2004, Muñoz, 2011).

Esta perspectiva generacional, con orígenes en la sociología propuesta por Karl Mannheim (1993), contribuye con lentes analíticos para observar los diversos ámbitos de producción de sociedad. Ella aporta algunas pistas epistémicas, por lo tanto, teóricas, políticas y de método, a las cuales recurrimos en la presente reflexión: 
i) por una parte, la conformación historizada de generaciones, que son variables en el tiempo y que coexisten en su diversidad, que permite enfrentar analíticamente la tendencia a la naturalización de las relaciones entre generaciones y de los conflictos que emergen entre ellas;

ii) por otra parte, lo generacional permite comprender la arbitrariedad de la asignación de características esenciales a sujetos jóvenes, adultos/as y otros/as, pasando más bien a análisis en que se considera su permanente recreación y los condicionamientos estructurales que se van produciendo en cada época;

iii) también, la conflictividad entre generaciones, además de ser desnaturalizada por la vía de la historización que ya señalamos, permite una comprensión de ella como relaciones de poder variables y contextuales - cada persona será considerada joven o viejo en referencia a alguien o a un cierto proceso social (Bourdieu, 1990) - en que se posibilita conceptualizar dicha conflictividad, así como las posibles alternativas para su enfrentamiento y superación;

iv) además, esta perspectiva generacional aporta elementos estructurales para comprender la constitución de un orden adultocéntrico que sostiene relaciones de dominio en diversos ámbitos de la sociedad; de igual manera, nos parece que la perspectiva generacional, así utilizada, nos permitirá plantear orientaciones para diseñar estrategias de acción en diversos campos en que lo colaborativo entre generaciones será un aspecto fundante de dichas estrategias (Duarte, 2015).

Tomando en consideración estos elementos es que nos parece que la escuela, como expresión del sistema educativo institucional, puede y requiere ser observada también como un ámbito social en el que se relacionan generaciones; de la misma manera que interactúan clases, género, razas, etc. En esas generaciones que se vinculan, la presencia de personas adultas y de un conjunto de características que les atribuyen 
-la adultez- constituye el eje de análisis del presente artículo y ha ido ganando espacio en nuestros intereses en la comprensión de lo social usando este lente generacional. Haciendo foco sobre las formas de expresión de esas relaciones, las experiencias de acción educativa en que hemos participado en el Núcleo de Investigación y Acción en Juventudes (2019), así como las investigaciones actuales (Duarte, Hernández y Palenzuela, 2019), evidencian que hay al menos tres asuntos contextuales e institucionales en que podría ser útil agudizar la mirada.

Un asunto relevante, que viene llamando la atención en nuestro país en el ámbito educativo, dice relación con la convivencia escolar. Desde la muerte de un estudiante, a manos de un compańero a fines de los noventa, hasta la ley de "Aula segura" recientemente dictada, se evidencia que se trata de un asunto que ha ido adquiriendo cada vez mayor complejidad para los diversos actores, en lo que refiere a formas de enfrentar su producción -la convivencia- y los modos de gestionarla, cuando mayormente desde la legislación y el diseńo de políticas se insiste en fórmulas represivas y punitivas, en especial contra los estudiantes. Al mismo tiempo que se focaliza en el tratamiento de relaciones entre niños, niñas y jóvenes, y poco esfuerzo se dedica a la relación entre personas adultas o entre estas y sus estudiantes. Las preguntas que surgen aquí nos remiten a los roles que han jugado las y los actores adultos de las comunidades educativas en esta gestión de la convivencia, en las formas de concebir y enfrentar la conflictividad en el espacio escolar y de qué manera esas personas adultas se despliegan en estas situaciones.

De igual forma, el diseño curricular, los estilos pedagógicos y las didácticas utilizadas, así como los mecanismos de evaluación, son otro asunto que ha estado institucionalmente en manos de las personas adultas de las comunidades educativas, que suelen no concebir a las y los jóvenes como actores que podrían aportar en su diseño e implementación. Esta dinámica ha permitido que, por largo tiempo, el control del proceso educativo esté solo en manos de las personas adultas que ejercen roles de docencia, negando posibilidades de participación 
sustantiva y genuina (Palma, 1998; Anderson, 2001; Escobar y Pezo, 2019) a niñas, niños y jóvenes signados como estudiantes. A partir de ello, se naturalizan estos roles, y de esa forma las relaciones de dominio que existen en ellas se invisibilizan, aceptándolas como una condición que no se puede modificar. Del mismo modo, se refuerza la noción de que las personas adultas saben y quienes juegan roles de estudiantes no poseen conocimiento ni capacidad de aporte en la gestión de su proceso educativo, por tanto, el aprendizaje se vuelve unidireccional, lo cual dificulta toda posibilidad de avanzar hacia un aprendizaje colaborativo y recíproco.

Otro asunto son los mecanismos e instancias de toma de decisiones en la dinámica educativa. Aparece como dado que la resolución última en cualquier asunto de la cotidianidad escolar la ha de asumir la persona adulta, pues es ella quien está garantizada para hacerlo. Uno de sus fundamentos, sostenido en el paradigma adultocéntrico que reifica el ciclo vital (Duarte, 2018), es que las personas adultas son responsables, maduras y poseen rasgos de personalidad y conducta que las legitiman para tomar esas decisiones y que nadie más en la escuela las posee. Sin embargo, cuando indagamos tratando de que las y los diversos actores señalen qué se entiende por responsabilidad o madurez, no existen claridades sobre a qué remiten, a pesar de ser utilizadas como argumentos para resolver situaciones de tensión o para explicarse su cotidianidad. Por ello nos atrevemos a señalar que más bien son artificios adultocéntricos que permiten sostener las asimetrías propias de este sistema de dominio. Así, estas decisiones son obra y atributo solo de quienes juegan esos roles adultos en el sistema educativo, haciendo que nińos, niñas y jóvenes queden desprovistos de dicha posibilidad -aunque ha de considerarse que, en una acción de manifiesta incoherencia adulta, se les insiste a las y los estudiantes en que "deben aprender a tomar decisiones y hacerse responsables de ellas"-; sin embargo, las que se vinculan a su cotidianidad educativa, no están dentro de esas posibilidades de ensayo.

En síntesis, nuestra preocupación investigativa apunta a que la adultez, en sus diversas expresiones y en las diferentes vías de 
construcción social, no es cuestionada ni interpelada en tanto tal en nuestra sociedad. En las experiencias educativas, cuenta con una legitimidad totalizante que no deja espacios a interrogaciones críticas. Sin embargo, nuestra apuesta es que se requiere pasar de su naturalización a su historización como categoría socialmente construida que demanda ser repensada e imaginada con horizonte de transformación social. La perspectiva generacional puede ser una contribución en esta tarea complejizando la experiencia educativa, toda vez que los vínculos entre actores educativos no solo están mediados por el rol o función que se cumple en ella, sino que también por el ordenamiento generacional que se establece en las relaciones y estructura educativas.

\section{PRODUCCIÓN TEÓRICA SOBRE LA ADULTEZ EN LA ESCUELA EN EL CONTEXTO DE UNA SOCIEDAD ADULTOCÉNTRICA}

Hemos señalado en este texto la existencia de un sistema de dominio que denominamos adultocentrismo. En tanto tal, refiere a un orden estructural e institucional que impone una noción de adultez como superioridad -respecto de otros momentos del ciclo vital-y, al mismo tiempo, como referencia y horizonte que todas las personas deben alcanzar (niños, niñas y jóvenes), mantener y ojalá no perder (personas adultas mayores) (Duarte, 2018). Este sistema construye asimetrías que se evidencian en unas condiciones de accesos y clausuras a bienes materiales y oportunidades políticas, según el cumplimiento de lo que se denominan tareas para el desarrollo de cada individuo; en estas tareas, las que corresponden a la adultez y a las personas adultas son consideradas esencialmente superiores respecto de las que se les imponen a los otros momentos del ciclo vital. También se materializan estas asimetrías en unos imaginarios simbólicos que, a partir de la reificación del ciclo vital como universal, compuesto de etapas sucesivas en el tiempo, y con definiciones en que las características esperadas 
de la adultez y las personas adultas son valoradas en tensión con la subvaloración de las esperadas en la niñez, juventudes y adultez mayor (Duarte, 2018). Finalmente, consideramos este sistema de dominio como uno que impone una gestión de las corporalidades y sexualidades que se sostiene sobre la cosificación de los cuerpos y deseos de nińos, nińas, jóvenes y adultos/as mayores, poniendo como eje de su despliegue la propiedad que tendrían sobre esos cuerpos, que les permitiría decidir sobre sus deseos, orientaciones y modos de relación (Duarte, 2018).

Al mismo tiempo, para el interés de este artículo tenemos en consideración que el adultocentrismo refiere a un paradigma que construye un conjunto de explicaciones compartidas en sociedad. Estas adquirieron durante el siglo XX fundamento desde diversas ciencias, en especial médicas, sociales y educativas, que las consolidaron como idea hegemónica que se cristalizó y contribuyó al fortalecimiento del adultocentrismo como sistema de dominio en el contexto capitalista (Duarte, 2018). Asimismo, ayudó al potenciamiento de la adultez como universal simbólico del dominio, en el que una de sus manifestaciones principales consiste en que su existencia -la adultez- no requiere mayor explicación y es inmanente en su presencia.

Así, en la cotidianidad educativa a menudo se emplean enunciados que dan cuenta de la importancia de la adultez dentro de la institución escolar; incluso, en ocasiones se apela a dicha noción para suscitar determinados comportamientos considerados como deseables. Así, por ejemplo, frases como: “Madure, compórtese como adulto/a!” vienen a dar cuenta de que lejos de ser un concepto anecdótico más bien se constituye como un elemento que está presente en los modos de relación que construyen los distintos actores educativos, y también en el propio ordenamiento de la estructura educacional como expresión de este adultocentrismo.

Sin embargo, ¿̇a qué nos referimos cuándo hablamos de ser adulto/a en la escuela? Al revisar el tratamiento de la adultez en la institución escolar es posible constatar que la producción investigativa y teórica resulta casi inexistente; ello en gran medida puede ser afectado, 
como ya señalamos, por el carácter natural que se le atribuye. De esta manera, pareciera que ser y/o hacerse adulto/a en el espacio educativo aparece como algo dado y no producido socialmente, de ahí que no genere mayor interés de ser considerado como un objeto de estudio. A contracorriente de esta idea, planteamos que preguntarse sobre los significados que se le atribuyen, lejos de considerarla como una pregunta esencialista que cristaliza su comprensión en un determinado entendimiento, posibilita abrir cuestionamientos genuinos para resituar su impronta como constructo social.

Ante ello, y a modo de movilizar su discusión, presentamos una revisión que da cuenta de dos perspectivas teóricas - contrapuestassobre la adultez, que develan implícitamente su configuración y su incidencia en la realidad educativa, aunque cabe insistir en que se trata de abordajes tangenciales en el campo educativo. Así, este ejercicio inicial pretende ofrecer un panorama que oriente y entregue pistas sobre sus miradas influyentes.

\section{a) La adultez como un operador funcional y normativo en la realidad educativa}

Esta vertiente constituye una visión optimista y positiva de entender la adultez, pues concibe que ella es fundamental dentro del quehacer educativo, constituyéndose como un modelo de autoridad necesario para desplegar los procesos de enseńanza-aprendizaje, posibilitando a su vez la transmisión de valores hacia las generaciones consideradas como menores y dependientes de ella. En ese sentido, los aportes provenientes de la sociología y la psicología han ejercido un papel preponderante para reforzar dicho imaginario.

Al respecto, Durkheim (1990), situado desde la sociología funcionalista, comprende que la educación constituye un mecanismo fundamental dentro de la socialización e integración social, posibilitada gracias a la acción ejercida por las generaciones adultas hacia las generaciones jóvenes que permite suscitar en estas un conjunto de estados físicos y mentales que se consideran como necesarios para la 
vida social. De ahí que la educación sea entendida como un medio para preparar e inculcar las condiciones de cada niño/a, así como también el funcionamiento de la sociedad en su conjunto. Dicho en términos del autor:

la educación es la acción ejercida por las generaciones adultas sobre aquellas que no han alcanzado todavía el grado de madurez necesario para la vida social. Tiene por objeto el suscitar y desarrollar en el nińo un cierto número de estados físicos, intelectuales y morales que exigen de él tanto la sociedad política en su conjunto como el medioambiente específico al que está especialmente destinado. (p. 47)

En sintonía con ese planteamiento siempre dentro de la sociología funcionalista, Parsons (1990) profundiza sobre el sentido de la educación como un órgano de socialización, el cual impartiría a estudiantes los conocimientos necesarios para el cumplimiento de sus obligaciones y también para el funcionamiento del orden social de manera eficaz. De este modo, la educación emanciparía gradualmente al niño de su apego inicial con la familia, inculcando a las nuevas generaciones valores y conocimientos necesarios para la vida social, diferenciándolos y jerarquizándolos según su rendimiento. Es decir, permite que "los alumnos internalicen los compromisos y habilidades necesarias para el desempeño feliz de sus futuros roles de adultos, y segundo, funciona para establecer la asignación de estos recursos humanos dentro de la estructura de roles de la sociedad adulta" (p. 32). A partir de ello, refuerza la idea de que la educación constituye un medio que orienta y prepara el camino hacia la adultez y, simultáneamente, enfatiza la importancia del rol del adulto-docente dentro del espacio educativo, otorgándole una superioridad y autoridad tanto en los conocimientos que posee sobre las asignaturas, como en la responsabilidad con la cual está investida dicha figura. Desde las experiencias concretas en que nos vinculamos con comunidades educativas, hemos encontrado que estas perspectivas teóricas, lejos de estar superadas, se encuentran plenamente vigentes en los imaginarios de las personas adultas, que 
se refieren a ellos cada vez que buscan argumentar/legitimar sus prácticas educativas y sus roles como adultos/as.

En cuanto a la adultez, para las perspectivas funcionalistas, esta se configura como un elemento central dentro del proceso educativo siendo importante tanto para la transmisión de ideas y valores a las nuevas generaciones, así como también para el ejercicio de autoridad. Además, se infiere que es justamente la institución escolar la que permite proyectar el futuro de las y los estudiantes, preparándoles para la vida adulta y para el funcionamiento en la vida social. No obstante, no queda del todo claro y resuelto qué es lo propio de la adultez o cuáles serían los roles sociales de esa adultez, careciéndose a su vez de una justificación empírica y/o científica que respalde dichos postulados. Ello no solo resulta relevante desde el plano lógico-argumentativo, sino también por su arbitrariedad, ya que tiende a reproducir imaginarios estigmatizadores pues, visto así, impide el reconocimiento de los estudiantes en el tiempo presente, dibujándolos solo como actores del mañana.

Por su parte, desde el campo de la psicología del desarrollo, diversas teorías han intentado contribuir a la comprensión del ciclo vital, aunque cabe mencionar que como denominador común mantienen una racionalidad lineal y evolutiva de las fases y/o etapas, y donde a cada una de ellas se le atribuye un conjunto de tareas que todo individuo debiese cumplir para alcanzar la plenitud del desarrollo, entendida esta dentro de la adultez (Krauskopf, 2010). De esta forma, y tal como señalamos, las otras etapas (niñez, juventud y adultez mayor) quedan dibujadas como incompletas. Lo anterior predominó en gran parte del siglo XX, cuando la preocupación se centró en las primeras etapas del desarrollo, invisibilizando lo que se entendía por adultez. Así, autores como Hall (1904) son pioneros en establecer una demarcación entre la adolescencia y la vida adulta, siendo retomado este tipo de análisis por la teoría del desarrollo psicosexual (Freud, 1958), la teoría del desarrollo psicosocial (Erikson, 1977) y las teorías psicoeducativas (Piaget, 1972), que centraron su preocupación en la teoría del aprendizaje y del desarrollo cognitivo. 
Este modo de comprender el ciclo vital se ha convertido en el paradigma predominante en el entramado social, y también dentro del campo educativo, teniendo gran influencia en los planes curriculares de formación pedagógica, constituyéndose como el imaginario reificado que circula socialmente (Duarte, 2016). De esta manera, la adultez por oposición o incluso por negación se ha convertido en la figura que orienta y conduce el devenir de los demás momentos del ciclo vital.

Ante ello, en el contexto contemporáneo diversas investigaciones (Ferrada, 2014; Hollmann et al., 2007; Prieto, 2008) vienen a reforzar este imaginario, señalando que la figura del adulto-docente tiene una impronta de formador y guía para las nuevas generaciones, siendo un modelo correcto que orienta a estudiantes, teniendo un doble propósito: educacional y social. Incluso, a pesar de que se reconozca que con poca frecuencia se habla del lugar del adulto frente a niños/as y jóvenes, se insiste en que son los sujetos considerados como menores quienes requieren de atención, cuidado y tutelaje, pues es deber de los actores adultos protegerlos y mantener cierta asimetría que permita la transmisión de conocimientos, valores culturales y normativos.

\section{b) La adultez como condensación de desigualdad generacional}

Esta vertiente releva una mirada crítica y/o negativa de comprender la adultez dentro del espacio escolar. Así, lo que se pone en discusión dentro de esta mirada es que los modos de relación que se construyen al interior de los espacios educativos suelen sostenerse por relaciones desiguales, conflictivas y fragmentadas. Si bien el énfasis está puesto en los vínculos que se erigen al interior de los establecimientos educacionales, de modo tangencial se releva la influencia que ejercen aquellos actores adultos en dichas dinámicas. Con relación a ello, es posible observar dos perspectivas que discuten sobre su configuración; la primera, centrada en la autoridad pedagógica de docentes, y la segunda, centrada en las relaciones generacionales. 
Desde el campo de la discusión de la autoridad pedagógica son variados los elementos y posturas que entran en discusión, no obstante, todas coinciden en que no es posible establecer una acción pedagógica sin el ejercicio de autoridad, ya sea reconociéndola como un elemento necesario para el correcto funcionamiento de la educación y la sociedad, o incluso para aquellas perspectivas críticas que dan cuenta de que la educación como violencia simbólica requiere de la autoridad pedagógica para reforzar y legitimar el poder arbitrario (Bourdieu y Passeron, 1977).

Con relación a ello, y situado desde lo que denominó una pedagogía sociologizada, Martucelli (2009) ahonda sobre el problema del ejercicio de autoridad en la escuela, reconociendo tres diagnósticos sobre sus desafíos en el contexto contemporáneo. Un primer diagnóstico da cuenta del debilitamiento del rol docente, debido a que en el contexto actual la autoridad ya no reposa sobre la institución o sistema, sino que depende de las propias capacidades individuales. De esta manera, si antes el orden escolar se basó sobre las capacidades coactivas, ahora cada docente debe poner en juego sus propios atributos o estilos para ejercer la autoridad, cuando más bien ello deriva de una fragilidad institucional que requiere de una gestión colectiva y solidaria de la indisciplina escolar.

Sobre este primer diagnóstico, llama la atención que dicho debilitamiento docente sea explicado únicamente por la pérdida del peso institucional, desconociendo la influencia del componente generacional que subyace en la cuestión de la autoridad, a pesar de que sea el mismo Martucelli quien también lo señale:

en el marco de la institución escolar y de una sociedad regulada por la tradición, la obediencia de los alumnos a la autoridad del profesor y de los adultos en general, y en primer lugar los padres, aparecían como pilares centrales del orden social. (Martucelli, 2009, p. 103)

Asimismo, si bien plantea un abordaje colectivo sobre el ejercicio de autoridad, esta es considerada como un atributo exclusivo para docentes, sin profundizar la justificación de ello. 
El segundo diagnóstico planteado por Martucelli (2009) refiere a la influencia de la evaluación escolar y la selección social, las cuales operan potencialmente como un mecanismo de desestabilización de la autoridad del docente, debido a que pueden afectar tanto las trayectorias sociales como la disciplina en las salas de clases. Finalmente, el tercer diagnóstico pone de relieve el componente generacional, señalando que la transformación de la cultura juvenil y de las relaciones de poder entre las generaciones ha removido la autoridad docente. Así, argumenta un cambio de las relaciones de fuerzas entre estudiantes y docentes, provocado por la legitimidad de la cultura juvenil que desea una mayor comunicación y un trato horizontal. Al respecto, cabe la duda de que este diagnóstico efectivamente dé por sentado un cambio en las relaciones de fuerzas entre adultos y jóvenes, pues justamente es ello lo que se pone en tensión desde la visión más crítica de la adultez. Asimismo, si bien se menciona una cultura juvenil y una cultura escolar, se extrańa alguna referencia hacia una cultura adulta, lo cual deja pendiente a explorar. Más bien observamos que, lejos de cuestionar el fundamento de la autoridad que reposa sobre el rol docente, mantiene anclada la idea de que ella es necesaria dentro del aparato educativo.

Así, ante la imposibilidad de renunciar a ella, las discusiones han girado en cómo comprenderla y los mecanismos que adopta dentro de la institución escolar. En ese sentido, es posible distinguir por un lado aquellas investigaciones que centran su preocupación en la diferenciación entre la autoridad y el autoritarismo (Reyes et al., 2018; Gallego et al., 2016; Guillot, 2006); la primera más bien respondería a aquella obediencia recíproca y voluntaria, mientras que la segunda referiría a un conjunto de prácticas impositivas, coactivas y violentas por parte de docentes hacia estudiantes. Por otro lado, se identifica aquellas investigaciones que, compartiendo la tesis del declive institucional que afecta a la autoridad, plantean la necesidad de avanzar en su legitimidad recíproca, toda vez que ella implica el reconocimiento y respeto mutuo entre docentes y estudiantes dentro del proceso pedagógico (Zamora et al., 2015; Neut, 2015). 
De esta forma, los estudios asociados a la autoridad dan cuenta de ciertas conflictividades, tensiones entre las relaciones de docentes y estudiantes, no obstante, ella tiende a ser ignorada en términos generacionales, siendo reducida también solo al vínculo entre ambos actores, lo que pierde de vista las relaciones y la convivencia en las que participa toda la comunidad educativa.

Desde una aproximación distinta, diversos estudios (Pezo, 2019; Vásquez, 2013; López-Moreno y Alvarado, 2011; Beheran, 2010; Duarte, 2002) han considerado la perspectiva generacional para dar cuenta críticamente de cómo se configuran las relaciones intergeneracionales entre mundos adultos y mundos juveniles en el espacio escolar. Dichas investigaciones comparten que los modos de relación suelen estar sostenidos por relaciones sociales asimétricas que mantienen los vínculos relacionales fragmentados y tensionados, lo que en gran medida ha sido conceptualizado como adultocentrismo. Si bien dicha clave conceptual ha sido utilizada como una noción que denuncia las desigualdades generacionales, no siempre se profundiza sobre su comprensión, tendiendo a ser equiparada a las lógicas disciplinarias de la escuela (Lay-Lisboa y Montañés, 2017; Vásquez, 2013). Ante ello, más bien hemos sugerido que el poder disciplinario se refuerza y complementa con el poder adultocéntrico (Pezo, 2019); de ahí la necesidad de distinguir y precisar su configuración, reconociéndolo a través de su triple dimensión: material, simbólico y corporal-sexual (Duarte, 2002, 2018).

A partir de esta revisión sucinta, ha de considerarse que tanto las perspectivas funcional/positiva como crítica/negativa relevan que la adultez produce una influencia en la institución escolar, aunque distan en el carácter que se le atribuye a dicha incidencia. Ante ello, consideramos que lejos de mantener este debate en términos dicotómicos, esta revisión pone de manifiesto al menos dos consideraciones que resultan medulares para este artículo. La primera, relevar la pertinencia de una impronta generacional para leer la experiencia educativa, y la segunda, invitar a problematizar una 
categoría que, pese a la importancia atribuida, ha sido invisibilizada como objeto de estudio.

\section{LA ESCUELA COMO LUGAR DE PERSONAS ADULTAS, PERO NO DE ADULTEZ}

Tal como hemos venido planteando, el sistema educativo como institución social generacional cuenta con personas adultas que juegan roles de dirección en su diseño y gestión. Dicha dinámica se encuentra en todos los ámbitos o niveles de este sistema, siendo la escuela nuestro lugar prioritario de preocupación en esta reflexión.

En el contexto de una sociedad adultocéntrica, que impone formas de dominio a partir de unas ciertas nociones de adultez como superioridad y, en el mismo movimiento, de niñez, juventud y vejez como inferioridad, es cada vez más urgente evidenciar el escaso tratamiento de esta adultez como una construcción social, a contracorriente de las ideas impuestas por siglos de que ella se constituye como resultado natural de los procesos de vida de cada individuo en la sociedad. Como hemos venido planteando, este artificio adultocéntrico consigue invisibilizar el peso simbólico que tiene esta adultez en las diversas instituciones sociales -familia, escuela, políticas públicas, medios de comunicación, entre otras-, reafirmando la asimetría en que se funda este sistema de dominio $y$, al mismo tiempo, otorgando a quienes juegan roles de mayoridad la capacidad de someter a quienes son posicionados como menores. Se les dota así a aquellos/as de las posibilidades para controlar los procesos en que se involucran y de sancionarlos en función de sostener las asimetrías adultocéntricas.

Los imaginarios adultocéntricos posicionan a la adultez como referencia a alcanzar y obedecer para niños, niñas y jóvenes; como pérdida para las personas adultas mayores, y en lo global como universal simbólico que cuenta con la ya señalada legitimidad para desenvolverse en sociedad. 
En las escuelas, estos modos de construcción de adultez se refuerzan con tres mecanismos que consolidan los marcos de actuación de las personas adultas. Por una parte, y como ya adelantábamos, se naturalizan las capacidades que cada actor posee en la jerarquizada distribución de roles y atributos. Esta naturalización consiste en atribuir a fuerzas sobrenaturales aquello que cada actor realiza y los argumentos que pueden explicar sus acciones. De esta manera, se neutraliza cualquier iniciativa de cambio o modificación de dichas acciones y discursos -y de los imaginarios que las sostienenporque basta la vinculación a aquel argumento natural para que se asuma que es incuestionable. Este mecanismo tiene un efecto: al ser considerados naturales los caracteres atribuidos a la adultez, se niega cualquier posibilidad de transformación por la vía de la acción humana, es decir son transformados en asuntos fatales. La adultez sería inmodificable, quizás en coherencia con lo que su etimología señala: "adulto es el que ya creció" (Corominas, 1987), vale decir, terminó su proceso de búsqueda propio del tiempo de juventud. Al autopercibirse como una persona con identidad definida, madurez alcanzada y con la responsabilidad como característica intrínseca, las y los adultos en la escuela tienden a cerrar cualquier posibilidad de ser interpelados por sus experiencias educativas cotidianas y, en las experiencias de acción directa que realizamos, hemos constatado dificultades para reconocer lo que podrían aprehender en la relaciones con sus estudiantes: se tiende a negar que existan asuntos por conocer y que posean carencias que las personas consideradas como menores podrían aportarles.

Otro mecanismo es la universalización de la adultez, como una referencia sin historia, sin tiempo, despegada de cualquier contexto, cultura, clase, género, etc. Es decir, se trataría de una noción homogeneizante que no muestra especificidades ni variaciones de acuerdo con los contextos en que se materializa. A partir de su no necesidad de cambio, sus características pasan a ser consideradas estables e inmutables en el tiempo, incluso respecto de personas adultas que viven en contextos muy diferentes, y que pertenecen a clase, géneros, 
razas y generaciones diversas. Es tan profunda la universalización de la adultez, que todo lo que podría ser parte de su identificación es tratado socialmente como una propiedad que no puede cambiar, que se alcanza y no se pierde: madurez, responsabilidad, sabiduría, razón, seriedad, entre otras. De esta forma, se vuelven atributos cristalizados que incluso las propias personas adultas no pueden controlar, porque están definidos desde fuera de ellas; sí pueden valerse de esos atributos para ganar prestigio y beneficios en su experiencia social, lo que nos lleva al tercer mecanismo.

Socialmente existe un conjunto de beneficios que las personas adultas consiguen, sostenidos sobre esta relación asimétrica de carácter adultocéntrico que hemos mencionado. Esta noción refiere al mecanismo de los privilegios de la adultez que nos interesa problematizar, en tanto resultado de las asimetrías de poder entre generaciones en la escuela, así como hipótesis del porqué las personas adultas no se movilizan por su transformación. La condición adulta provee de estos privilegios, como ventajas ante las demás personas: para imponer ideas a sus estudiantes, definir formas de acción y relación en la institución educativa, controlar las decisiones en los distintos ámbitos de la vida de la comunidad educativa. El privilegio mayor, en el ámbito de las relaciones entre generaciones en la escuela, es no ser cuestionado, organizar la cotidianidad a su antojo y recibir beneficios que refuerzan su posición de autoridad. Reconocemos en nuestra experiencia que la escuela ha mutado y que han variado las expresiones de las relaciones generacionales, sin embargo, las distribuciones de poder en ella y la distribución en las relaciones de fuerzas se siguen reproduciendo a partir de lógicas adultocéntricas; es decir, ha mutado, pero no se ha transformado.

Estos mecanismos de consolidación y reproducción de los imaginarios de adultez en la escuela requieren ser problematizados e investigados en profundidad, para contribuir a que las relaciones al interior de las comunidades educativas se desplieguen en un sentido democrático y, al mismo tiempo, quienes juegan roles de adultez puedan reelaborar los mandatos adultocéntricos impuestos. Esta 
producción de conocimientos aportará al cuestionamiento del orden adultocéntrico construido y a imaginar alternativas a él.

\section{APERTURA REFLEXIVA}

En este último apartado, en continuidad con lo ya planteado, queremos proponer al menos tres hipótesis de trabajo que podrían dar pie al inicio de procesos investigativos en comunidades educativas y/o a procesos de acción directa en escuelas. Por ello, más que cierre, lo concebimos como una apertura a nuevas oportunidades de producción de saberes que pongan en tensión el orden adultocéntrico y provean de alternativas para su transformación.

Hemos sostenido la importancia y necesidad de estudiar la adultez, en tanto su construcción social responde a un cierto orden de poder asimétrico que se reproduce en el dominio. Se trata en ese sentido de un concepto acientífico, que no ha sido debatido en profundidad ni de forma sistemática (Duarte, 2015). De igual manera, nos parece que no se trata de un concepto descriptivo, sino de una noción que condensa una conflictividad social latente que queda invisibilizada en su posición de privilegio. En ese sentido, su estudio podría aportar a la densificación de este, en tanto expresión de unas dinámicas sociales que requieren de atención.

Por ello, tal como señalamos previamente, nuestro propósito es una apuesta por visibilizar dicha conflictividad y avanzar en las construcciones de alternativas. Como hemos sugerido, el análisis con la perspectiva generacional como estrategia puede ser de gran provecho para las y los actores de las comunidades educativas; de igual forma, como episteme de los análisis que se implementen en procesos de producción de conocimientos. Desde ambos procesos, o en la confluencia de ellos, los diálogos intergeneracionales pueden ser una base fundamental para ensayar la necesaria colaboración entre generaciones en nuestra sociedad. 
A continuación, presentamos tres asuntos que nos parecen relevantes de atender como posibles líneas de investigación y de acción. Usamos el formato de hipótesis de trabajo, en tanto posibles explicaciones para ciertos nudos problemáticos en la realidad que queremos observar, y en tanto guías para dichos análisis comprensivos. No son enunciados por verificar o falsear en la acción comunitaria y en la reflexión crítica que en ella realizamos.

\subsection{Imaginarios, discursos y prácticas de la adultez en la institución escolar}

¿Por qué es pertinente preguntarse cómo se construye la adultez en la escuela? Esta pregunta contiene desde ya una afirmación que, como hemos sostenido, refuerza la idea de que la adultez - en todas sus variantes de clase, género, generación, territorio y raza- se constituye como una construcción en cada sociedad y cultura, en cada época. Pues bien, cautelamos no asumir la pregunta en un modo que podría arrastrarnos a un cierto esencialismo; nos interesa más bien agudizar nuestro foco de observación sobre los mecanismos sociales y las dinámicas en que esas diversas formas de adultez se construyen en la escuela.

Para llevar adelante este ejercicio nos parece fundamental poner en discusión los diversos estereotipos que sobre esa adultez se producen cotidianamente -mediante los mecanismos ya debatidos de naturalización y cristalización-, en especial en los modos de reproducción a través de discursos y prácticas.

También podemos abordar la pregunta anterior mirando la relevancia política de asumir este desafío. Ello nos lleva a hipotetizar que este develamiento podría ayudar a las y los actores de la escuela y a la institución que constituyen a construir nuevas formas de adultez, en concordancia con nuevos propósitos en los procesos educativos, con adultos/as colaborativos/as, respetuosos/as y solidarios/as, es decir, permitiría ensayar otros modos de relación. 


\subsection{Vínculos entre la adultez y los procesos de enseñanza- aprendizaje}

¿Cuál es el tipo de adultez que se requiere para procesos de enseñanzaaprendizaje liberadores? En esta interrogante nos ubicamos desde un tipo de educación, aquella que concibe a las y los diversos actores presentes en ella como sujetos con capacidades y potencialidades para contribuir en su despliegue y ganar en autonomía y autoestima en dicho proceso. Nos ubicamos desde una escuela, y un sistema educativo, que se funda sobre el fortalecimiento de la agencia de cada actor/a. Por ello, abordar esta pregunta exige tensionar los métodos de aprendizaje tradicional-bancarios para avanzar en procesos dialógicos de aprendizaje. En este sentido, el diálogo de saberes -con características de horizontalidad y democracia- se instituye como condición fundante de otra escuela.

Si bien serían múltiples los asuntos que podríamos debatir en este ámbito, nos parece que cuestionar los modos de construir lo que se denomina la autoridad pedagógica y sus fundamentos es una cuestión que se vuelve de alta relevancia. Se trata de ir más allá de la sola observación de los mecanismos de su ejercicio; más bien, lo que se requiere es preguntarse por quiénes pueden ejercer su autoridad y qué adultez se requiere para que todas las personas involucradas puedan hacerse parte del ejercicio de control del proceso educativo.

En este sentido, la consideración de lo generacional nos parece clave, en tanto nos permite centrar la mirada en las condiciones de las relaciones que se verifican en los espacios educativos. Desde este foco, podemos potenciar la noción de equivalencia de roles generacionales en la escuela, en que se otorgue importancia semejante a quienes, desde sus distintas posiciones institucionales, aportan también saberes diferenciados en el proceso. Ahora bien, esa diferencia no tendría por qué convertirse en desigualdad o asimetría, sino en diversidad en tanto se respetan y valoran los diferentes aportes que cada cual realiza.

Lo que buscamos entonces es interrogar por las condiciones que habría que forjar en la escuela para ensayar procesos de simetría de poder entre generaciones, para construir otra escuela, principalmente 
una en que se comparta el control de los procesos educativos y de las tomas de decisión, desafiándose las y los distintos actores a superar las lógicas de simulacros que priman en estas relaciones.

\subsection{La adultez en los modos de convivencia escolar}

¿Por qué la convivencia entre personas adultas en la escuela es un asunto invisible y poco abordado en la investigación y en la acción educativa? ¿Qué aportaría a las comunidades educacionales, y a quienes las componen, analizar y actuar sistemáticamente en la convivencia entre estas personas adultas? Hemos evidenciado antes que los modos de relación entre personas adultas reproducen conflictividades diversas, que no son atendidas ni visibilizadas como tales. Incluso, en nuestra experiencia de acción directa con las comunidades educativas, hemos constatado que las y los adultos en ocasiones reconocen cierta incoherencia o contradicción en sus prácticas, pues si bien promueven y exigen un buen trato entre estudiantes, este imperativo no logra ser observado y garantizado hacia toda la comunidad educativa donde se incluya su participación como actores adultos, lo que también es observado ante las miradas de estudiantes.

La clave generacional podría ser de mucha ayuda en esta interrogante, dado que permitirá observar al cuerpo docente, directivo y de asistentes de la educación -las personas consideradas adultas en la escuela-como un conjunto de generaciones adultas que se interrelacionan, también desde sus diversas experiencias y sentidos propios. Por ejemplo, en los procesos que hemos acompańado, vemos que en la superficie de la conflictividad la diferencia de edad aparece como el factor explicativo principal; sin embargo, a medida que profundizan en su autoobservación, van complejizando factores y, por ejemplo, entre docentes el tipo de formación recibida en su formación universitaria parece ser una marca relevante: profesores/as normalistas, docentes con licenciatura en universidades post reforma neoliberal, quienes hicieron su formación en cursos abreviados, docentes que cuentan además con posgrados en la materia, entre otras modalidades. Todo 
ello los ubica con unas experiencias diversificadas que los ponen en posiciones distintas al momento de desplegarse en el quehacer cotidiano de la escuela, tomando formas de acción también distintas. Esa diferenciación no es necesariamente vista como un aporte de complementariedad y sinergia, sino que es causal de tensión que corroe las relaciones y precariza el ambiente de trabajo.

El análisis de esa conflictividad y no su evasión es el asunto que nos interesa problematizar, a través de interrogar la cultura adulta que se evidencia en la cultura escolar. Nos parece que podría ser un aporte a las comunidades educativas poner de manifiesto su existencia y lo que se ganaría con su tratamiento específico para la convivencia escolar democrática.

\section{REFERENCIAS}

Álvarez, C. (2018). La perspectiva generacional en los estudios de juventud: enfoques, diálogos y desafíos. Última Década, 26(50), 40-60. https://dx.doi.org/10.4067/S0718-22362018000300040

Anderson, G. (2001). Hacia una participación auténtica: deconstrucción de los discursos de las reformas participativas en educación. En Nuevas tendencias en politicas educativas: estado, mercado y escuela (pp. 145-200). Ediciones Juan Granica.

Beheran, M. (9 y 10 de diciembre de 2010). Relaciones intergeneracionales y escolaridad en contextos de desigualdad social. En VI Jornadas de Sociología de la UNLP, La Plata, Argentina. Universidad Nacional de La Plata. Facultad de Humanidades y Ciencias de la Educación. Departamento de Sociología. http://sedici.unlp. edu.ar/handle/10915/104978

Bourdieu, P. (1990). La juventud no es más que una palabra. En Sociología y cultura. Grijalbo. 
Bourdieu, P., Passeron, J. C., Melendres, J., y Subirats, M. (1977). La reproducción: elementos para una teoría del sistema de enseñanza. Laia.

Corominas, J. (1987). Breve diccionario etimológico de la lengua castellana. Gredos.

Duarte, K. (2002). Mundos jóvenes, mundos adultos: lo generacional y la reconstrucción de los puentes rotos en el liceo. Última Década, 10(16): 95-113. http://dx.doi.org/10.4067/S071822362002000100004

Duarte, K. (2015). El adultocentrismo como paradigma y sistema de dominio. Análisis de la reproducción de imaginarios en la investigación social chilena sobre lo juvenil [tesis de doctorado]. Universidad Autónoma de Barcelona. https://www.tdx.cat/handle/10803/377434

Duarte, K. (2018). Genealogía del adultocentrismo. La constitución de un patriarcado adultocéntrico. En Duarte, C. y Álvarez, C. (Eds.). Juventudes en Chile. Miradas de jóvenes que investigan (vol. 2). Social-Ediciones.

Duarte, K., Hernández, N. y Palenzuela, Y. (2019). Juventudes en Chile: miradas de jóvenes que investigan II. Social-Ediciones.

Durkheim, E. (1990). Educación y sociología. Península.

Erikson, E. (1977). Identidad, juventud y crisis. Paidós.

Escobar, S. y Pezo, H. (2019). Más allá del concepto: experiencias y reflexiones en torno a la participación juvenil estudiantil. Última Década, 27(52), 65-79. https://dx.doi.org/10.4067/S071822362019000200065

Ferrada Rau, R. N. (2014). Relación entre docentes y estudiantes. Una indagación a las representaciones sociales que construyen estudiantes de educación media en torno a la influencia de la institución escolar en sus procesos formativos [tesis de magíster]. Universidad de Chile. http://repositorio.uchile.cl/handle/2250/134843 
Freud, A. (1958). Adolescence. The Psychoanalytic Study of the Child, (13)1, 255-278 https://doi.org/10.1080/00797308.1958.11823182

Gallego, L., Acosta, J., Villalobos, Y., López, A. y Giraldo, A. (2016). Violencia del docente en el aula de clase. Revista de Investigaciones UCM, 16(28), 116-125. http://www.revistas.ucm.edu.co/ojs/index. $\mathrm{php} /$ revista/article/viewFile/81/81

Ghiardo, F. (2004). Generaciones y juventud: una relectura desde Mannheim y Ortega y Gasset. Última Década, 12(20), 11-46. https://dx.doi.org/10.4067/S0718-22362004000100002

Guillot, G. (2006). La autoridad en la educación. Salir de la crisis. Popular.

Hall, S. (1904). Adolescence. Its Psychology and Its Relations to Physiology, Anthropology, Sociology, Sex, Crime, Religion and Education. Appleton \& Company.

Hollmann, J., García, M. y Lerner, M. (2007). El lugar de los adultos frente a los niños y los jóvenes. Aportes para la construcción de la comunidad educativa. Buenos Aires: Ministerio de Educación, Ciencia y Tecnología de la Nación. http://repositorio.educacion. gov.ar/dspace/handle/123456789/94812

Krauskopf, D. (2010). La condición juvenil contemporánea en la constitución identitaria. Última Década, (18)33, 27-42. http:// dx.doi.org/10.4067/S0718-22362010000200003

Lay-Lisboa, S. y Montañés, M. (2017). ¿Escuela adultocéntrica, contraadultocéntrica, exoadultocéntrica, academicista? La infancia nos habla de relaciones transformadoras en el espacio educativo, International Journal of Sociology of Education, 6(3), 323-349. http://dx.doi.org/10.17583/rise.2017.2500 
López-Moreno, L. y Alvarado, S. Emergencia de las relaciones intergeneracionales en una escuela pública urbana. Revista Latinoamericana de Ciencias Sociales, Niñez y Juventud, 1(9), pp. 255-268. http://biblioteca.clacso.edu.ar/Colombia/alianza-cindeumz/20130711013311/ArtLigiaLopezmoreno.pdf

Mannheim, K. (1993). El problema de las generaciones. REIS: Revista Española de Investigaciones Sociológicas, 62, 193-244. https:// dialnet.unirioja.es/servlet/articulo?codigo $=766796$

Martuccelli, D. (2009). La autoridad en las salas de clase. Problemas estructurales y márgenes de acción. Diversia, 1, 99-128. https:// docer.com.ar/doc/ne8vle

Muñoz, V. (2011). Generaciones. Juventud universitaria e izquierdas politicas en Chile y México (Universidad de Chile - UNAM 19842006). LOM Ediciones.

Neut, P. (2015). La autoridad pedagógica desinvestida y la reconfiguración de sus legitimidades instituyentes. Revista de la Academia, (19), 59-96. https://doi.org/10.25074/0196318.0.17

Núcleo de Investigación y Acción en Juventudes (2019). Caja de Herramientas. Participación Juvenil en el Liceo. Mineduc.

Palma, D. (1998). La participación y la construcción de ciudadanía. Universidad ARCIS, Centro de Investigaciones Sociales.

Parsons, T. (1990). El salón de clase como sistema social: algunas de sus funciones dentro de la sociedad norteamericana. Revista de Educación. (242), 64-86. http://hdl.handle.net/11162/71526

Pezo, H. (2019). ¿Liceo disciplinario?: condicionantes para la participación juvenil estudiantil. Una aproximación desde la perspectiva generacional. En K. Duarte, N. Hernández y Y. Palenzuela, Juventudes en Chile: miradas de jóvenes que investigan II (pp. 286-312). Social-Ediciones.

Piaget, J. (1972). De la lógica del niño a la lógica del adolescente. Paidós. 
Prieto, E. (2008). El papel del profesorado en la actualidad. Su función docente y social. Foro de Educación, 6(10), 325-345. https:/dialnet. unirioja.es/servlet/articulo?codigo $=2907073$

Reyes, J. J. M., González, C. B. L., y Corona, A. C. (2018). Ser docente: entre la autoridad y el autoritarismo. RECIE. Revista Electrónica Cientifica de Investigación Educativa, 4(1), 753-761. https:/www.rediech.org/ojs/2017/index.php/recie/article/view/307

Vásquez, J. (2013). Adultocentrismo y juventud: Aproximaciones foucaulteanas. Sophia, 1(15), 218-234. https://doi.org/10.17163/ soph.n15.2013.08

Viveros, M. (2016). La interseccionalidad: una aproximación situada a la dominación. Debate Feminista, 52, 1-17. https://doi. org/10.1016/j.df.2016.09.005

Zamora-Poblete, G., Meza-Pardo, M. y Cox-Vial, P. (2015). ¿De dónde surge la autoridad de los profesores chilenos? Análisis desde las perspectivas de los estudiantes. Magis, Revista Internacional de Investigación en Educación, 7(15), 63-80. https://dialnet.unirioja. es/servlet/articulo?codigo $=5014388$ 